\title{
Pretreatment of industrial phosphoric acid, removal of major impurities: Modeling and optimization
}

\author{
Y. Bounou ${ }^{1}$, D. Zakaria ${ }^{1}$ and K. Ibno Namr ${ }^{2}$ \\ ${ }^{1}$ Laboratoire de Physico-Chimie des Matériaux (LPCM), Département de Chimie \\ 2 UR Sciences du sol et environnement (LGMSS - URAC 45), Département de Géologie Université Chouaïb \\ Doukkali, Faculté des Sciences, BP. 20, 24000 El Jadida, Maroc
}

\begin{abstract}
Modeling by full factorial design is used to determine the optimal operating conditions for the pretreatment of the industrial phosphoric acid from sulfate, fluoride, organic matter, chromium and cadmium. The effects of process factors (sediment weight ratio to phosphoric acid, temperature and time reaction) on the removal of these impurities were determined and correlated to the mechanism of their elimination. The results predicted using linear model show high values of regression coefficients $\left(\mathrm{R}^{2}\right.$ sulfate $=0.97, \mathrm{R}^{2}$ fluoride $=0.97, \mathrm{R}^{2}$ organic matter $=0.95, \mathrm{R}^{2} \mathrm{Cr}=0.96$ and $\mathrm{R}^{2} \mathrm{Cd}=0.86$ ) indicating good agreement with experimental data. Optimization was carried out by using desirability function by targeting the factors to desired levels for maximum removal of these impurities. The efficiencies of removal of sulfate, fluoride, organic matters, chromium and cadmium were $63 \%, 81 \%, 78 \%, 15 \%$ and $19 \%$ respectively, with a global desirability of 0.75 .
\end{abstract}

\section{INTRODUCTION}

Wet process phosphoric acid is produced by a chemical reaction of the phosphate rock with a mineral acid. The obtained wet phosphoric acid (WPA) contains a number of undesirable ionic impurities originally present in the phosphate rock, like sulfate, fluorine, organic matters and metals. Such acid can be used directly in fertilizers manufacture. For others uses, such as, food industries and phosphates derivatives, phosphoric acid must be purified. On an industrial scale one proceeds generally by a preliminary treatment to decrease the rate of the major impurities: sulfates, fluoride, organic matter and heavy metals. In this study, we used natural sediment, based on dolomite, clay (illite) and silica which allowed an encouraging result to pre-treat phosphoric acid. In addition to the category of material, several factors can influence pre-treatment WPA process, such as, dose of sediment to phosphoric acid weight ratio, temperature and reaction time. Modeling and optimization of this process were carried out using designs of experiment (DOE).

\section{MATERIAL AND METHODS}

\subsection{Sediment for phosphoric acid pretreatment}

The natural sediment used in this study was mined at a depth of 3 meters from Doukkala's region subsoil (Morocco). Sediment was crushed and sieved less than $100 \mu \mathrm{m}$. It was selected to investigate the pretreatment process of WPA in order to profit from the advantages of its abundance and its low cost comparing to the solvents and chemical reagents. This study is novel in a way as no literature citations are available where this sediment has been used for phosphoric acid pretreatment. The pretreatment protocol was judiciously chosen in order to simulate the industrial conditions. The experiments of pretreatment were carried out in a polyethylene reactor with a mechanical stirrer. Phosphoric acid was heated to the desired temperature, then the quantity of sediment was added and the mixture was stirred at the desired time. The values for these three factors were fixed by full factorial design (FFD) methodology of DOE. The total mixture was filtered off from the phosphoric acid.

\subsection{Instrumentation}

Sulfate $\left(\mathrm{SO}_{4}^{2-}\right)$ was carried out by ionic chromatography (ICS_3000, column IonPac_AS19_Analytical_4*250mm, detector-conductimeter, eluent $\mathrm{KOH})$. Fluoride $\left(\mathrm{F}^{-}\right)$was determined by potentiometric method (JENCO model 6209). Organic matter (OM) was determined by Walky and Black method. Chromium $(\mathrm{Cr})$ and cadmium $(\mathrm{Cd})$ were carried out by ICP-ES (Jobin Yvon ULTIMA 2). Solid recovered was characterized by X-ray diffraction (XRD) (Philips X'Pert PRO diffractometer with $\mathrm{CuK} \alpha$ radiation $\lambda=1.5418 \AA$ ).

\subsection{Statistical DOE by FFD}

DOE was used to study the effect of the important variables in the phosphoric acid pretreatment by natural sediment. FFD allows the simultaneous study of the effects that several factors may have on the pretreatment process optimization. It determines which factors have the important effects on the response as well as how the effect of one factor varies with the level of the other factors. The effect of a factor is defined as the change in response produced by a change in the level of the factor. This is frequently called a main effect because it refers to the primary factors of interest in the experiment [1]. In the contrast of the "one variable a time" approach, FFD allows measuring the interaction between different factors. In the present study, two-level FFD is used for the modeling the phosphoric acid pretreatment 
Table 1. FFD matrix with observed (Yobs.), predicted (Ypred.) responses (ppm) and regression coefficient $\mathrm{R}^{2}$.

\begin{tabular}{|c|c|c|c|c|c|c|c|c|c|c|c|c|c|c|c|c|}
\hline \multirow[b]{2}{*}{ run } & \multicolumn{3}{|c|}{ Codified values } & \multicolumn{3}{|c|}{ True values } & \multicolumn{2}{|c|}{$\mathrm{SO}:{ }_{4} \mathrm{R}^{2}=0.975$} & \multicolumn{2}{|c|}{$\mathrm{F}^{-}: \mathrm{R}^{2}=0.972$} & \multicolumn{2}{|c|}{$\mathrm{Cd}: \mathrm{R}^{2}=0.86$} & \multicolumn{2}{|c|}{ Cr: $R^{2}=0.957$} & \multicolumn{2}{|c|}{ OM: $\mathrm{R}^{2}=0.95$} \\
\hline & $\mathrm{X}_{1}$ & $\mathrm{X}_{2}$ & $\mathrm{X}_{3}$ & $\mathrm{X}_{1}$ & $\mathrm{X}_{2}$ & $\mathrm{X}_{3}$ & $\mathrm{Y}_{\text {obs. }}$ & $\mathrm{Y}_{\text {pred. }}$ & $\mathrm{Y}_{\text {obs. }}$ & $\mathrm{Y}_{\text {pred. }}$ & $\mathrm{Y}_{\text {obs. }}$ & $\mathrm{Y}_{\text {pred. }}$ & $\mathrm{Y}_{\text {obs. }}$ & $\mathrm{Y}_{\text {pred. }}$ & $\mathrm{Y}_{\text {obs. }}$ & $Y_{\text {pred. }}$ \\
\hline 1 & -1 & -1 & -1 & 1 & 20 & 10 & 11567 & 11465 & 1454 & 1487 & 34 & 35 & 460 & 453 & 60 & 59.95 \\
\hline 2 & 1 & -1 & -1 & 5 & 20 & 10 & 8120 & 8018 & 2034 & 2068 & 40 & 41 & 657 & 650 & 44 & 43.95 \\
\hline 3 & -1 & 1 & -1 & 1 & 80 & 10 & 10206 & 10104 & 816 & 850 & 36 & 37 & 640 & 633 & 118 & 117.95 \\
\hline 4 & 1 & 1 & -1 & 5 & 80 & 10 & 7730 & 7628 & 1515 & 1548 & 38 & 39 & 568 & 561 & 29 & 28.95 \\
\hline 5 & -1 & -1 & 1 & 1 & 20 & 30 & 8563 & 8461 & 1995 & 2029 & 42 & 43 & \begin{tabular}{|l|}
769 \\
\end{tabular} & 762 & 59 & 58.95 \\
\hline 6 & 1 & -1 & 1 & 5 & 20 & 30 & 10497 & 10395 & 2281 & 2315 & 40 & 41 & 562 & 555 & 63 & 62.95 \\
\hline 7 & -1 & 1 & 1 & 1 & 80 & 30 & 7554 & 7452 & 621 & 654 & 36 & 37 & 582 & 575 & 31 & 30.95 \\
\hline 8 & 1 & 1 & 1 & 5 & 80 & 30 & 4992 & 4890 & 785 & 819 & 52 & 53 & 735 & 728 & 15 & 14.95 \\
\hline 9 & 0 & 0 & 0 & 3 & 50 & 20 & 7736 & 8552 & 1739 & 1471 & 46 & 40 & 625 & 615 & 52 & 52.33 \\
\hline
\end{tabular}

process by natural sediment. Among their advantages is the reduction in the number of required experiments, resulting in lower reagent consumption, less time laboratory work and modeling the process for determining the significance of each factor.

\section{RESULTS AND DISCUSSION}

\subsection{Modeling by FFD}

Application of DOE requires the adequate selection of responses, factors and their levels. As is known, FFD has optimum properties to estimate a linear model as shown in the following equation (2):

$$
\begin{aligned}
& Y=b_{0}+b_{1} X_{1}+b_{2} X_{2}+b_{3} X_{3}+b_{12} X_{1} X_{2} \\
& +b_{13} X_{1} X_{3}+b_{23} X_{2} X_{3}+b_{123} X_{1} X_{2} X_{3}+\varepsilon
\end{aligned}
$$

where $Y$ is the concentration of impurities (response to fit and to optimize), $X_{1}, X_{2}$ and $X_{3}$ (factors in codified units), $b_{0}$ is the global mean, $b_{i}$ and $b_{i j}$ are the measures of the effect of variables $X_{i}$ and $X_{i} X_{j}$ respectively. The variable $X_{i} X_{j}$ represents the first order interaction between $X_{i}$ and $X_{j}(i<j)$ and $\varepsilon$ is the random error associated with the response. The responses selected $(Y)$ are: sulfate, fluoride, organic matter (OM), chromium and cadmium. The selection of factors $(X)$ was made considering the chemistry of the system (to be adapted to the available industrial process conditions) and the practical use of the FFD. The factors affecting the pretreatment process are: dose of natural sediment to phosphoric acid weight ratio $\left(X_{1}\right)$, reaction temperature $\left(X_{2}\right)$ and reaction time $\left(X_{3}\right)$. The working pressure was hold at $760 \mathrm{~mm} \mathrm{Hg}$ and the stirring speed was set to $600 \mathrm{rpm}$. Selection of the levels was carried out on the basis of the results obtained in the industrial scale and for economic reasons. Table 1 shows the range of process variables and the standard experimental matrix for the FFD together with data for observed (Yobs.) and predicted (Ypred.) responses.

The main $\left(b_{i}\right)$ and the interaction $\left(b_{i j}\right)$ effects of each factor having p-values $<0.05$ are considered as potentially significant. The analysis of variance (ANOVA) for the linear model (Eq. (2)) was carried out to establish its significance. The adjusted $\mathrm{R}^{2}$ was well within acceptable limits of $\mathrm{R}^{2} \geq 0.8$ [1]. After estimating the

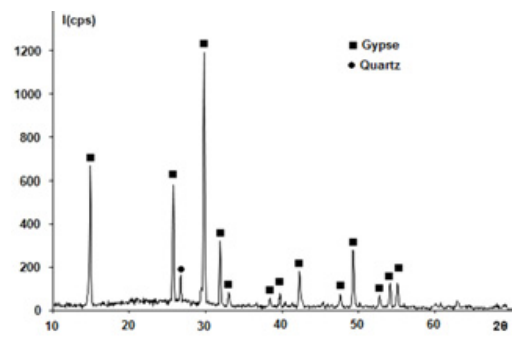

Figure 1. XRD for the sediment recovered after WPA pretreatment.

main and interacting effects, the factors affecting the removal of impurities were determined by performing the statistical test for each effect (Table 2). The significance of each factor in the process is quantified by the $\mathrm{p}$-value, its importance increase as the $\mathrm{p}$ value decreases [2].

For sulfate removal, the effects of all factors were significant. The increase of temperature, time and dose of sediment facilitates the removal of sulfate. The factor "dose" interacts strongly with temperature and time, indicating a synergetic influence in process removal. Sulfates are precipitate as gypsum by chemical reaction with dolomite (Figure 1).

For fluoride removal, the main effects of temperature and reaction time are statistically significant. For the interaction effects, only interaction dose-time has a high statistical significance, de-fluoration is done by reaction of fluoride with silica or with aluminum to form a volatilized compounds $\mathrm{H}_{2} \mathrm{SiF}_{6}$ [3] or $\mathrm{AlF}_{6} \mathrm{MgNa}, 6 \mathrm{H}_{2} \mathrm{O}$ (Ralsonite) [4] and it is favorable while the heating and time contact (under stirring) were as high as possible. For OM removal, the main and the interactions effects are significant, sediment dose and time have an important effect in the elimination by adsorption on the gypsum formed [5]. Cadmium and chromium were retained by adsorption on gypsum. As shown in Figure 2, the isotherm adsorption is type III or type $\mathrm{S}$ [6], where $\mathrm{Q}_{e}$ and $\mathrm{C}_{e}$ are the concentrations of $\mathrm{Cr}$ and $\mathrm{Cd}$ in solid and phosphoric acid respectively. Dose of sediment has less importance than time and temperature; all the interactions effects are significant.

For the statistical model fitted, the coefficient of determination $\left(\mathrm{R}^{2}\right)$, which is a measure of degree of fit, was at least $R^{2}>0.86$. Therefore, the developed model 
Table 2. main $\left(b_{i}\right)$ and interaction $\left(b_{i j}\right)$ effects for the responses $\left(\mathrm{Y}_{i} \mathrm{ppm}\right)$.

\begin{tabular}{|l|r|r|r|r|r|r|r|r|r|r|}
\hline & \multicolumn{2}{|c|}{$\mathbf{S O}_{\mathbf{i}}$} & \multicolumn{2}{|c|}{$\mathbf{F}^{-}$} & \multicolumn{2}{c|}{ OM } & \multicolumn{3}{|c|}{ Cr } & \multicolumn{2}{c|}{ Cd } \\
\hline $\mathbf{b}_{\mathbf{i}}$ & \multicolumn{1}{|c|}{ Estimate } & \multicolumn{1}{|c|}{$\mathrm{p}$ value } & Estimate & $\mathrm{p}$ value & \multicolumn{1}{c|}{ Estimate } & $\mathrm{p}$ value & Estimate & $\mathrm{p}$ value & Estimate & $\mathrm{p}$ value \\
\hline $\mathrm{b}_{0}$ & $1.2 \times 10^{6}$ & 0.00 & 1471 & 0.00 & 52.33 & 0.00 & 621.6 & 0.00 & 39.8 & 0.00 \\
\hline $\mathrm{b}_{1}$ (Dose \%) & $-3.9 \times 10^{5}$ & 0.02 & 216 & 0.20 & -14.62 & 0.00 & 8.9 & 0.08 & 2.8 & 0.06 \\
\hline $\mathrm{b}_{2}$ (Temperature $\left.{ }^{\circ} \mathrm{C}\right)$ & $-5.3 \times 10^{5}$ & 0.02 & -503 & 0.03 & -4.12 & 0.02 & 9.6 & 0.07 & 0.8 & 0.01 \\
\hline $\mathrm{b}_{3}$ (time $\left.\mathrm{mn}\right)$ & $-4.7 \times 10^{5}$ & 0.02 & -17 & 0.02 & -10.37 & 0.00 & 40.4 & 0.02 & 2.8 & 0.00 \\
\hline $\mathrm{b}_{12}$ & $-2.4 \times 10^{5}$ & 0.03 & 0 & 0.13 & -11.62 & 0.00 & 11.4 & 0.06 & 1.8 & 0.02 \\
\hline $\mathrm{b}_{13}$ & $4.1 \times 10^{5}$ & 0.02 & -104 & 0.07 & 11.62 & 0.00 & -22.4 & 0.03 & 0.8 & 0.03 \\
\hline $\mathrm{b}_{23}$ & $3.6 \times 10^{5}$ & 0.04 & -214 & 0.04 & -14.87 & 0.00 & -13.1 & 0.05 & 0.8 & 0.02 \\
\hline $\mathrm{b}_{123}$ & $-4.1 \times 10^{5}$ & 0.02 & -30 & 0.07 & 6.62 & 0.01 & 78.6 & 0.00 & 2.8 & 0.01 \\
\hline
\end{tabular}
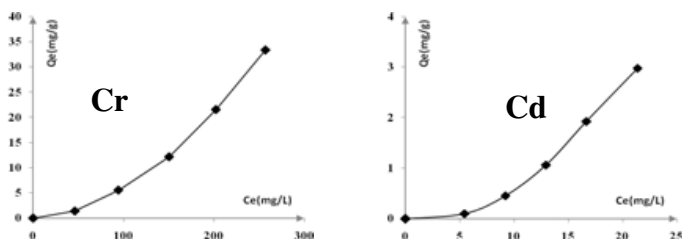

Figure 2. Adsorption isotherm for $\mathrm{Cr}$ and $\mathrm{Cd}$.

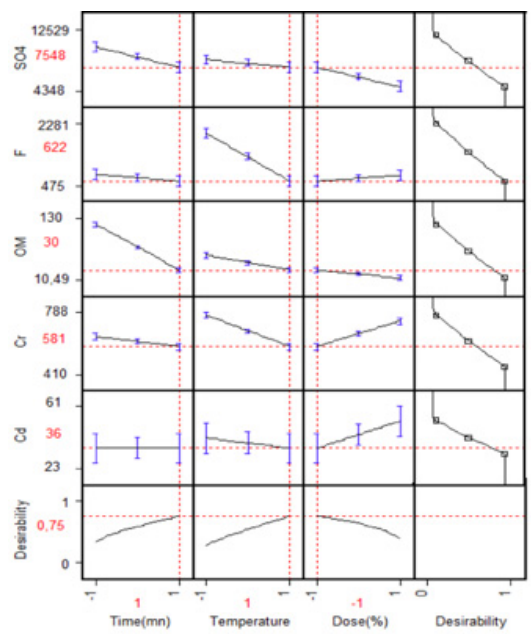

Figure 3. Desirability profiler plot for $\mathrm{SO}_{4}^{2-}, \mathrm{F}^{-}, \mathrm{OM}, \mathrm{Cr}$ and $\mathrm{Cd}$.

could adequately represent the real relationship among the factors chosen.

\subsection{Optimization study}

The optimum operating conditions for the pretreatment process were carried out by using the desirability function embedded in JMP-SAS software. For optimizing simultaneously the elimination of all impurities the operating conditions are: dose $=1 \%$, temperature $=80^{\circ}$ $\mathrm{C}$ and time $=30 \mathrm{mn}$ (Figure 3). Because of the difference in the mechanism of elimination, under these optimal conditions, the elimination efficiencies for sulfate $(63 \%)$, fluoride $(81 \%), \mathrm{OM}(78 \%)$ are great than those for chromium (15\%) and cadmium (19\%). Indeed, sulfate and fluoride are removed by chemical reaction while metals by adsorption. These results are obtained without taking account the water evaporation, which induces an increase in the concentrations of all chemical elements, particularly the phosphor.

\section{CONCLUSION}

The use of this sediment (dolomite, illite and silica) for WPA pretreatment allows a remarkable decrease in the rate of major impurities (sulfate, fluoride, OM, chromium and cadmium) to the industrial accepted limit for fertilizers application and purification process. FFD was used to investigate optimal operating conditions for WPA pretreatment. Desirability function was applied to target the factors to the desired levels for optimizing simultaneously the impurities removal. The experimental protocol proposed can be used in the industrial scale.

The authors wish to thank the Pole of Competence National Network of Physical Chemistry of Materials (REMAT) for financial support to realize this work.

\section{References}

[1] T. Lundstedt, E. Seifert, L. Abramo, B. Thelin, A. Nyström, G. Pettersen, R. Bergman, Chemometr. Intell. Lab. Syst. 42 (1998).

[2] R. Gottipati, S. Mishra, Chem. Eng. J. 160 (2010).

[3] Z. Qafas, K. El-Kacemi, E. Ennaassia, M. Chahib, Sci. Lett. 3 (3) (2002).

[4] P. Becker, Phosphates and Phosphoric Acid, Raw Materials, Technology and Economics of the Wet Processes, Dekker, New York (1989).

[5] M.A. Rauf, Shahnaz M. Qadri, Sarmadia Ashraf, Karima M. Al-Mansoori, Chem. Eng. J. 150 (2009).

[6] C.H. Giles, T.H. MacEvan, T.H.S.N. Nakhawa, D. Smith, J. Chem. Soc. 4 (1960). 